\title{
A CASE REPORT OF CUTANEOUS MENINGIOMA: UNCOMMON TUMOUR AT THE UNCOMMON REGION
}

\author{
(1) Egemen ISITAN ${ }^{1}$, (1) Esra UÇARYILMAZ ÖZHAMAM², (1) Aydın Talat BAYDAR ${ }^{3}$, (1) Servet GÜRECCCi², \\ ๑ Özhan Merzuk UÇKUN³ , ๑ Ali DALGIÇ3
}

\author{
${ }^{1}$ Erbaa State Hospital, Clinic of Neurosurgery, Tokat, Turkey \\ ${ }^{2}$ Ankara City Hospital, Clinic of Pathology, Ankara, Turkey \\ ${ }^{3}$ Ankara City Hospital, Clinic of Neurosurgery, Ankara, Turkey
}

\begin{abstract}
Meningiomas are usually located in the central nervous system and arise from the membranes surrounding the brain and spinal cord. Rarely, they may also be located in the skin and in this case they are known as cutaneous meningiomas. Herein, we report a case of cutaneous meningiomas within the dermal sinus tracts, located in the midline of the lumbosacral junction, the diagnosis of which was confirmed by histopathological examination.
\end{abstract}

Keywords: Cutaneous meningioma, epidermoid tumour, dermal sinus tracts, surgery

\section{INTRODUCTION}

Meningiomas are benign and slowly growing tumours that originate from arachnoid cap cells, which are located on the outer surface of the arachnoid membrane surrounding the brain and spinal cord ${ }^{(1)}$. They are usually located in the central nervous system (CNS) and tend to occur in any region within the brain and spinal cord(2). Rarely, they may also be located in the skin and are referred to as cutaneous meningiomas(3). These tumours originate from ectopic meningothelial cells in the dermis and subcutaneous tissue ${ }^{(3,4)}$. In addition, they may be found in the skin of patients with neural tube closure defects associated with congenital spinal malformations.

Here, we report a case of cutaneous meningioma within the dermal sinus tracts, located in the midline of the lumbosacral junction, the diagnosis of which was confirmed by histopathological examination.

\section{CASE REPORT}

A 35-year-old man presented with a 6-month history of low back and right leg pain. During physical examination, we found dermal sinus tracts with surrounding hyperpigmentation and hypertrichosis in the midline of the lumbosacral junction. His neurological examination was normal. To determine the aetiology of the pain, radiological studies were done, which showed a lumbar spinal lesion. In addition, magnetic resonance imaging (MRI) revealed an intra-dural/intra-medullary spaceoccupying lesion extending from the $\mathrm{L} 1-\mathrm{L} 4$ vertebrae and a dermal sinus tract in the midline of the lumbosacral junction (Figure 1).

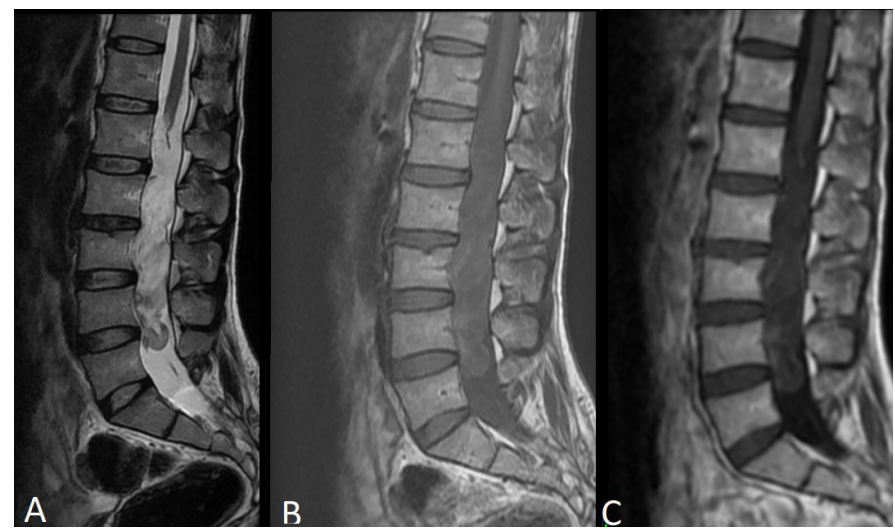

Figure 1. Pre-operative MRI scans revealed an intra-dural cystic lesion between L1 and L5 segments (A) lesion has no gadolinium enhancement according to the T1 and (B) T1+ contrast (C) scans. Dermal sinus tract is depicted using arrows in the lumbosacral junction as observed in FAST STIR MRI scan

FAST: Focussed assessment sonography for trauma, STIR: Short tau inversion recovery, MRI: Magnetic resonance imaging 
turkishspine

The patient was operated on in the prone position. The dermal sinus tract was dissected from the surrounding soft tissues
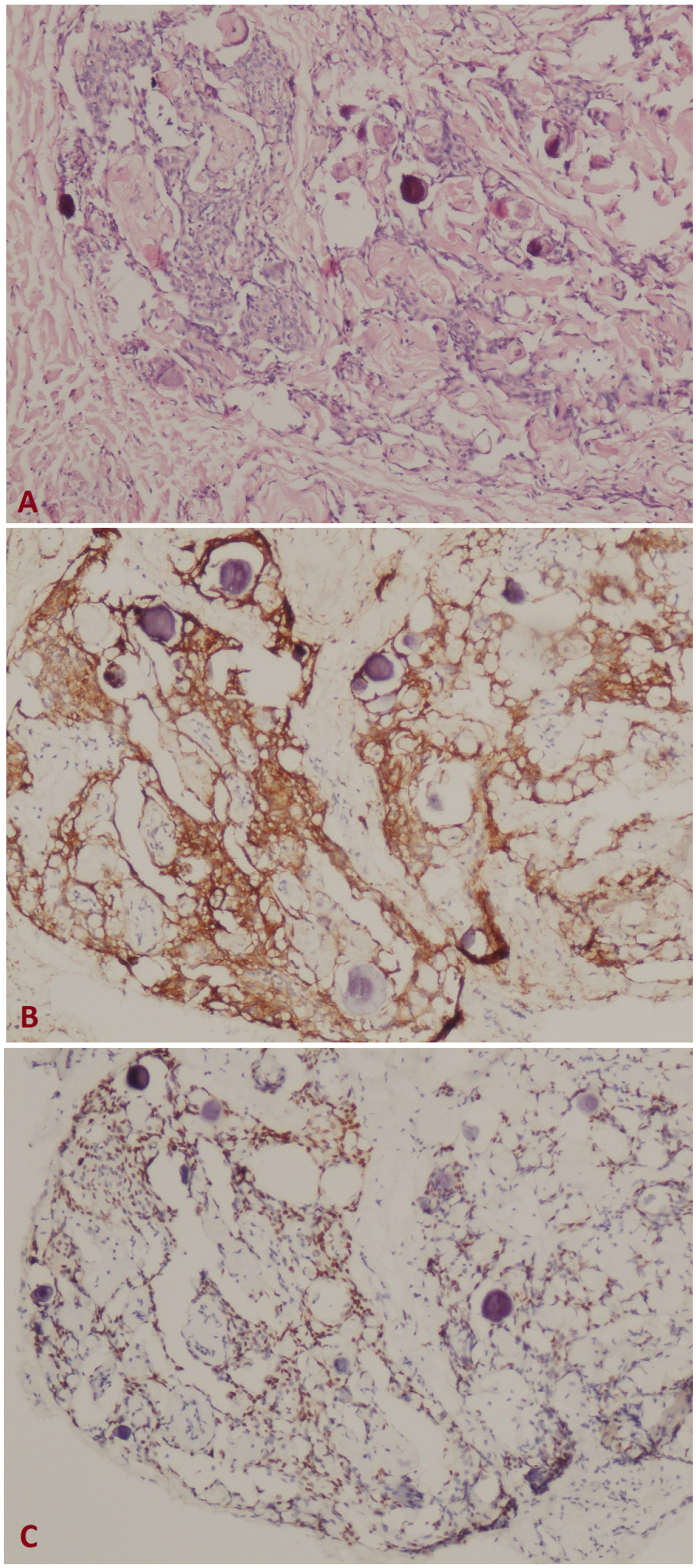

Figure 2. The meningothelial tumour cells are uniform, with oval nuclei, delicate chromatin and eosinophilic cytoplasm. There are whorls and psammoma bodies in the tumour (A). It stains for EMA (B) and progesterone receptor (C)

EMA: epithelial membrane antigen with a fish-mouth incision involving the tract, and the incision was extended to the L1 vertebra. The tract was first excised and then the intra-dural tumour was also excised via L1-L2-L4 hemilaminectomies. The tumour contained cutaneous elements such as hair. Upon discharge, the neurological examination for the patient was normal. The post-operative histopathological examination revealed that the intra-dural tumour contained keratinous materials (dermoid tumour) and that the fistula tract consisted of epithelial tissue and also a cutaneous meningioma with psammomatous calcifications (Figure 2). Post-operative MRI scans showed that the dermal sinus tract had been removed; however, a remnant of the dermoid tumour had persevered (Figure 3). The patient was followed-up for three years and during his last examination, mild numbness was detected in his right leg.

\section{Discussion}

Cutaneous meningioma was first reported in an Englandbased study ${ }^{(5)}$. In 1974, Lopez et al. ${ }^{(4)}$ classified these tumours using clinical and histopathological criteria. According to this classification, there are three distinct types of cutaneous meningiomas:

Type 1 cutaneous meningiomas are congenital and are discovered at an older age. They are mostly located in the scalp and paravertebral region, and they originate from ectopic arachnoid cells found between the dermis and subcutaneous layer during embryological development. These tumours may have a connection with the CNS in the form of sinus tracts which may be associated with neural tube defects like meningocele and meningomyelocele without the connection being interrupted ${ }^{(4,6)}$.

Type 2 cutaneous meningiomas originate from ectopic arachnoid cells that extend along spinal and cranial nerves and have no connection with the neural axis. These tumours mostly occur around sense organs, such as ears, nose and eyes ${ }^{(3,4)}$.

Type 3 cutaneous meningiomas occur as a result of the extension of intra-cranial meningiomas into the subcutaneous and dermal layers through bone and skin defects that develop after trauma or surgery ${ }^{(4)}$.

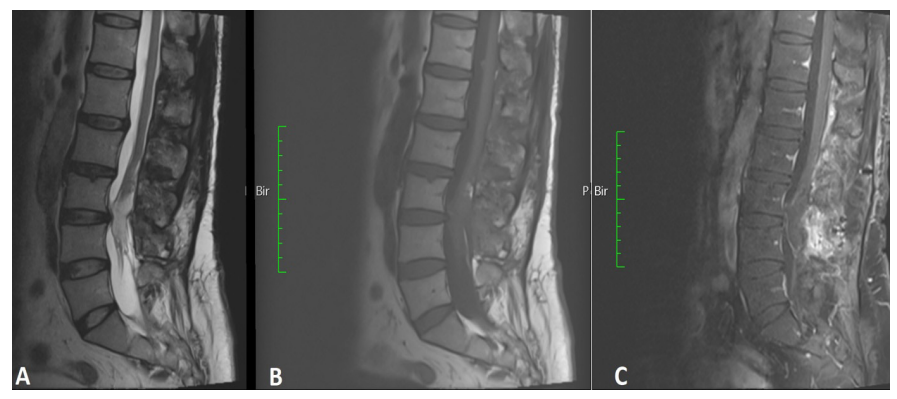

Figure 3. MRI scans showed that the epidermoid cyst lesion was removed sub-totally while the dermal sinus tract was removed totally $(\mathbf{A}, \mathbf{B}, \mathbf{C})$

MRI: Magnetic resonance imaging 
The differential diagnosis of cutaneous meningioma is generally difficult, because they are rare and have non-distinctive characteristic features such as painless subcutaneous nodules and hairless areas or hypertrichosis ${ }^{(3,4,7)}$. The differential diagnosis should include sebaceous nevus, alopecia areata, dermoid cysts, fibromas, haemangiomas, squamous cell carcinomas, hamartomas, meningocele, myelomeningocele, nasal gliomas, neuroectodermal tumours and metastatic lesions $s^{(3,4,6,7)}$.

It should be noted that type 1 cutaneous meningiomas may be associated with congenital tumours, such as epidermoid tumours or lipomas, and atypically located meningiomas in patients with congenital closure defects. Type 1 tumours have a better prognosis than type 2 and 3 tumours and they can be cured by en-bloc excision ${ }^{(3)}$. However, conservative treatment may be an option in selected patients who are asymptomatic or have a high morbidity burden for surgery ${ }^{(8)}$.

The patient in this study underwent surgery in adulthood after being diagnosed with a cystic tumour in the lumbar region and dermal sinus tracts in the lumbosacral junction. Clinical and histopathological findings were consistent with a type 1 cutaneous meningioma. Complete surgical resection is the gold standard treatment in such cases; thus, the tumour was removed completely from the dermal sinus tract in this case. In conclusion, meningiomas are common tumours of the CNS and their diagnosis is easy if the tumour occurs in the cranium and/or spinal canal; however, their diagnosis may be complicated when they are atypically located. In addition, because cutaneous meningiomas may occur in the skin, dermis and subcutaneous tissue, the differential diagnosis is important to avoid misdiagnosis. Cutaneous meningiomas should be considered in patients with spinal dysraphism or congenital tumours.
Ethics

Informed Consent: Informed consent was taken.

\section{Authorship Contributions}

Surgical and Medical Practices:A.D.,E.I.,Concept:A.D.,E.I.,Ö.M.U., Design: A.D., E.I., A.T.B., Data Collection or Processing: S.G., E.U.Ö., E.I., Analysis or Interpretation: S.G., E.U.H., Literature Search: E.I., A.T.B., Ö.M.U., Writing: A.D., E.I., S.G.

Conflict of Interest: No conflict of interest was declared by the authors.

Financial Disclosure: The authors declared that this study received no financial support.

\section{REFERENCES}

1. Mazloom SE, Holliday AC, Coman GC, Chavan RN, Kolodney MS, Grider DJ. Type I Cutaneous Meningioma (Rudimentary Meningocele) With Intradural Attachment to the Phylum Terminale. Am J Dermatopathol. 2016;38:927-9.

2. Kishore $M$, Kaushal $M$, Bhardwaj $M$, Sharma $N$. Cutaneous Meningioma: A Cytomorphological Diagnosis. Indian Dermatol Online J. 2017;8:201-4.

3. Miedema JR, Zedek D. Cutaneous meningioma. Arch Pathol Lab Med. 2012;136:208-11.

4. Lopez DA, Silvers DN, Helwig EB. Cutaneous meningiomas - a clinicopathologic study. Cancer. 1974;34:728-44.

5. Bain GO, Shnitka TK. Cutaneous meningioma (psammoma): report of a case. AMA Arch Derm. 1956;74:590-4.

6. Borggreven PA, de Graaf FH, van der Valk P, Leemans CR. Posttraumatic cutaneous meningioma. J Laryngol Otol. 2004;118:228-30.

7. Hussein MR, Abdelwahed AR. Primary cutaneous meningioma of the scalp: a case report and review of literature. J Cutan Pathol. 2007;34:26-8.

8. Campbell BA, Jhamb A, Maguire JA, Toyota B, Ma R. Meningiomas in 2009: controversies and challenges. Am J Clin Oncol. 2009;32:73-85. 